\title{
Multiscale Modeling and Simulation of Directional Solidification Process of Ni-Based Superalloy Turbine Blade Casting
}

\author{
Qingyan Xu * , Cong Yang, Hang Zhang, Xuewei Yan, Ning Tang and Baicheng Liu \\ Key Laboratory for Advanced Materials Processing Technology (Ministry of Education), School of Materials \\ Science and Engineering, Tsinghua University, Beijing 100084, China; yangc15@mails.tsinghua.edu.cn (C.Y.); \\ zhanghangmu@hotmail.com (H.Z.); yanxi920@126.com (X.Y.); tangn08@mails.tsinghua.edu.cn (N.T.); \\ liubc@tsinghua.edu.cn (B.L.) \\ * Correspondence: scjxqy@tsinghua.edu.cn; Tel.: +86-10-6279-5482
}

Received: 29 June 2018; Accepted: 8 August 2018; Published: 10 August 2018

\begin{abstract}
Ni-based superalloy turbine blades have become indispensable structural parts in modern gas engines. An understanding of the solidification behavior and microstructure formation in directional solidified turbine blades is necessary for improving their high-temperature performance. The multiscale simulation model was developed to simulate the directional solidification process of superalloy turbine blades. The 3D cellular automaton-finite difference (CA-FD) method was used to calculate heat transfer and grain growth on the macroscopic scale, while the phase-field method was developed to simulate dendrite growth on the microscopic scale. Firstly, the evolution of temperature field of an aero-engine blade and a large industrial gas turbine blade was studied under high-rate solidification (HRS) and liquid-metal cooling (LMC) solidification processes. The varying withdrawal velocity was applied to change the curved mushy zone to a flat shape. Secondly, the grain growth in the aero-engine blade was simulated, and the grain structures in the starter block part and the spiral selector part in the HRS process were compared with those in the LMC process. The simulated grain structures were generally in agreement with experimental results. Finally, the dendrite growth in the typical HRS and LMC solidification process was investigated and the simulation results were compared with the experimental results in terms of dendrite morphology and primary dendritic spacing.
\end{abstract}

Keywords: directional solidification; multiscale simulation; microstructure evolution; superalloy blade

\section{Introduction}

Nickel-based superalloy blades [1-7], which possess excellent high-temperature creep resistance, have been proven to be successful in aero-engine and industrial gas turbines (IGTs). To improve the high-temperature performance of superalloy blades, the columnar polycrystal and single crystal structures are favored, while the directional solidification (DS) techniques have become the choice for production over the past few decades. The most commonly used DS technique is the Bridgman technique [1], which was proposed by Bridgman and Stockbarger, and it has become the foundation of modern DS techniques. After that, high-rate solidification (HRS) [2] and liquid-metal cooling (LMC) [3] DS techniques were developed with an enhanced ability to provide a high temperature gradient, and they are widely used both in industrial production and experimental investigations. In the HRS technique, the temperature gradient is established by the heating zone and the cooling zone, while the main heat transfer approach involves the radiation between the ceramic mold and the furnace wall. In contrast, the LMC method has significantly improved the cooling efficiency by utilizing the liquid 
metal as the cooling medium and thus, higher temperature gradients can be obtained and a faster withdrawal velocity can be used. The high thermal gradient is favored in directional solidification, because it can reduce dendritic spacing [4], refine $\gamma^{\prime}$ morphology [5], and inhibit casting defects, such as freckle chains. However, the complex heat transfer process in the modern directional solidification process requires more precise control. Otherwise, defects, such as misaligned grains, stray grains, and freckles, can occur.

The mechanical properties of nickel-based superalloy blades are closely related with the casting microstructure, and an understanding of the formation of typical microstructure features is vital for improving its high-temperature performance. In recent years, numerical methods have emerged as a powerful tool in heat transfer computation and microstructure evolution simulation during alloy solidification. On the macroscopic scale, Gandin [8] and Kermanpur [9] proposed a cellular automaton-finite element (CA-FE) model for predicting grain structure in solidification, and the algorithm was applied to simulate columnar polycrystal and single crystal grain growth in directionally solidified turbine blades. Elliott $[10,11]$ used the CA-FE method to investigate the thermal profile and grain growth in the HRS and LMC DS processes, before comparing the mushy zone and grain morphology with experimental results. Zhang et al. [12-14] created a cellular automaton-finite difference (CA-FD) model to simulate the temperature evolution and grain growth in DS turbine blades, before developing a ray-tracing method to calculate the radiation. In the microscale simulation of superalloy dendrite growth, two major methods are used: the cellular automaton (CA) method [15] and the phase-field method [16-18]. Compared with the CA method, the phase-field [18] method uses scalar fields to represent the existence of different phases, and the evolution of each phase is based on the minimization of the local free energy. By coupling this with the superalloy thermodynamic database, the phase-field method is able to simulate the dendrite growth in multicomponent superalloy solidification [19]. The major disadvantage of the phase-field method is the large amount of calculation. Recently, the graphics processing unit (GPU) was used to accelerate the scientific computation, which allows for the realization of the phase-field simulation of large-scale 3D dendrite growth in directional solidification $[20,21]$.

In the current study, the multiscale modeling and simulation of directional solidified nickel-based superalloy blade were investigated. The heat transfer model [12,13], grain growth model [14,22,23], and multiscale coupling model [12,24], which were previously developed by our group, were summarized. On the macroscopic scale, the finite difference method was used to calculate the heat transfer process, and a modified CA method was used to simulate the grain growth. The developed CA-FD algorithm was used to simulate directional solidification of a single crystal blade in HRS and LMC processes. The temperature field distribution, mushy zone evolution, and grain structure of the two processes were compared and analyzed. After this, the algorithm was applied to optimize the withdrawal velocity in the LMC process for a large polycrystal industrial gas turbine blade. On the microscopic scale, the developed GPU-based phase-field method was used to simulate the dendrite growth in typical HRS and LMC directional processes. The dendrite morphology evolution and primary dendritic spacing under the different solidification conditions were obtained and analyzed.

\section{Methods}

Different models on different spatial scales are introduced in this section. The heat transfer model is used for simulating temperature evolution on the macro scale. The grain growth and dendrite growth model are used for simulating grain and dendrite growth on the micro scale. The multiscale coupling model is used for data transfer between the macro scale grids and the micro scale grids.

\subsection{Heat Transfer Model}

Figure 1 shows the schematic of the two DS techniques. In these two techniques, the mold shell and the casting move downwards from the heating zone to the cooling zone with the withdrawal unit. In the HRS process, the heat transfer mode between the mold shell and the furnace is radiation. 
In contrast, in the LMC process, the heat transfer mode changes from radiation to convective heat transfer as the shell mold descends into the liquid metal coolant. Therefore, the radiation angle factor between the mold and furnace elements changes during the withdrawal process, while there are also changes in the convective boundary conditions in the LMC process. The heat transfer model of the two DS techniques is given as follows.
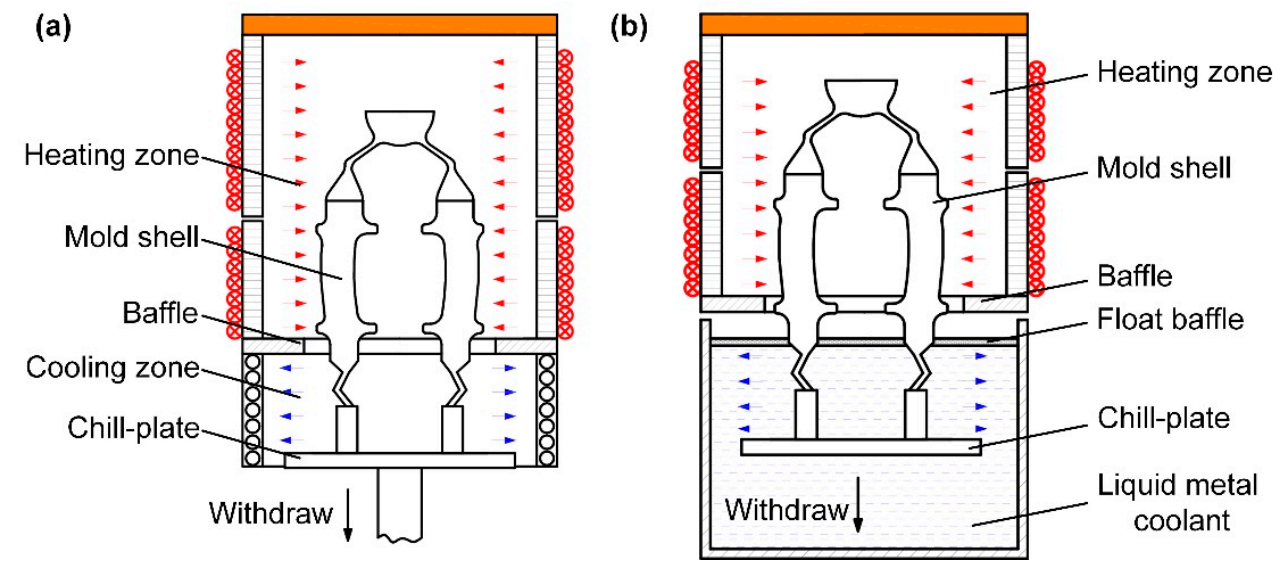

Figure 1. Schematic of the two types of directional solidification techniques: (a) HRS and (b) LMC.

The heat transfer process in directional solidification can be described by the law of the conservation of energy, which is written as:

$$
\rho c_{\mathrm{p}} \frac{\partial T}{\partial t}=\nabla \cdot(\lambda \nabla T)+Q_{\mathrm{M}}+Q_{\mathrm{R}}
$$

where $T$ is the temperature; $t$ is the time; $\rho$ is the material density; $c_{\mathrm{p}}$ is the material specific heat; $\lambda$ is the conductivity; $Q_{M}$ is the volumetric heat source; and $Q_{R}$ is the heat flux density between surface elements and the environment. When solidification happens, the liquid alloy releases latent heat and the volumetric heat source can be expressed as:

$$
Q_{\mathrm{M}}=\rho \Delta H \frac{\partial f_{\mathrm{s}}}{\partial t}
$$

where $\Delta H$ is the latent heat and $f_{\mathrm{s}}$ is the volume fraction of the solid phase. The heat flux density changes in different directional solidification techniques. A modified Monte Carlo-based ray tracing model [13] was used to calculate the heat radiation between the current element and the $n$th reference element as:

$$
Q_{\mathrm{R}}=\sigma \sum_{n=1}^{N} \frac{\beta_{n}\left(T^{4}-T_{n}^{4}\right)}{\frac{1-\varepsilon}{\varepsilon}+\frac{\left(1-\varepsilon_{n}\right) S}{\varepsilon_{n} S_{n}}+1},
$$

where $\sigma=5.67 \times 10^{-8} \mathrm{~W} \cdot \mathrm{m}^{2} \cdot \mathrm{K}^{-4}$ is the Stefan-Boltzmann constant; $N=50$ is the number of ray lines; $\beta_{n}$ is the energy factor; $T, \varepsilon$, and $S$ are the temperature, blackness coefficient, and surface area, respectively, of the current element; and $T_{n}, \varepsilon_{n}$, and $S_{n}$ are the reference temperature, blackness coefficient, and surface area, respectively, of the $n$th element. The heat flux density by convective heat transfer in the LMC process can be expressed as:

$$
Q_{\mathrm{R}}=h\left(T-T_{\mathrm{Ref}}\right)
$$

where $h$ is the convective heat transfer coefficient and $T$ and $T_{\text {ref }}$ are the temperatures of the current element and the reference element, respectively. 


\subsection{Grain and Dendrite Growth Model}

To simulate grain growth in superalloy solidification, a modified CA method [8,25] was used. A continuous nucleation model [25] was adopted to calculate the total nucleation density $n(\Delta T)$ at a given undercooling $\Delta T$ as:

$$
n(\Delta T)=\frac{n_{\max }}{\sqrt{2 \pi} \Delta T_{\sigma}} \int_{0}^{\Delta T} \exp \left[-\frac{1}{2}\left(\frac{\Delta T-\Delta T_{N}}{\Delta T_{\sigma}}\right)^{2}\right] d(\Delta T),
$$

where $n_{\max }$ is the maximum nucleation density; $\Delta T_{\sigma}$ is the standard deviation of undercooling; and $\Delta T_{N}$ is the mean nucleation undercooling. The grain growth speed $v(\Delta T)$ during solidification was calculated based on the Kurz-Giovanola-Trivedi (KGT) [26] kinetic equation:

$$
v(\Delta T)=\alpha \cdot \Delta T^{2}+\beta \cdot \Delta T^{3},
$$

where $\alpha$ and $\beta$ are kinetic coefficients.

The superalloy dendrite growth was simulated using the phase-field method [17], which has been widely applied in predicting microstructure evolution during alloy solidification. In the directional solidification, a frozen temperature approximation [27] is adopted, while the time evolution of the temperature is simplified as:

$$
T(z)=T_{0}+G\left(z-V_{\mathrm{p}} t\right),
$$

where $T_{0}$ is the temperature at $z=0 ; G$ is the temperature gradient obtained from the macroscopic heat transfer simulations; $V_{\mathrm{p}}$ is the withdrawal velocity; and $t$ is the time. The dimensionless phase-field kinetic equation and the solute transfer equation are given as:

$$
\begin{gathered}
\tau A^{2}(\vec{n}) \frac{\partial \phi}{\partial t}=\nabla\left(|\nabla \phi|^{2} A(\vec{n}) \nabla A(\vec{n})\right)+\nabla\left(A(\vec{n})^{2} \nabla \phi\right) \\
+\phi-\phi^{3}-\lambda\left(1-\phi^{2}\right)^{2}(U+\theta), \\
{\left[\frac{1+k-(1-k) \phi}{2}\right] \frac{\partial U}{\partial t}=\nabla} \\
\cdot\left(D_{l} \frac{1-\phi}{2} \nabla U+\frac{1}{2 \sqrt{2}}(1+(1-k) U) \frac{\partial \phi}{\partial t} \frac{\nabla \phi}{|\nabla \phi|}\right) \\
+\frac{1}{2}[1+(1-k) U] \frac{\partial \phi}{\partial t},
\end{gathered}
$$

where $\phi$ is the phase-field variable; $\theta=\left(T-T_{M}-m c_{\infty}\right) /|m| c_{\infty}(1 / k-1)$ is the dimensionless undercooling, where $T_{M}$ is the melting temperature of the pure solvent; $m$ is the liquidus slope; $c_{\infty}$ is the initial concentration; and $k$ is the partition coefficient. Furthermore, $U=\left(c_{l}-c_{l}^{e}\right) /\left(c_{l}^{e}-c_{s}^{e}\right)$ is the dimensionless concentration, where $c_{l}$ is the liquid solute concentration; $c_{l}^{e}$ and $c_{S}^{e}$ are the equilibrium concentration in the liquid and solid, respectively. $D_{l}$ is the solute diffuse coefficient in the liquid. Furthermore, $\lambda=a_{1} W_{0} / d_{0}$ is the coupling constant which determines the conversion factor between the dimensionless length and the dimensional length, where $a_{1}=0.8838, W_{0}$ is the phase-field interface width, and $d_{0}$ is the chemical capillary length. In directional solidification, the relaxation time is $\tau=\tau_{0}[1-(1-k) \theta]$, where $\tau_{0}=a_{2} \lambda W_{0}^{2} / D_{l}$ and $a_{2}=0.6267$. The 3D crystal anisotropy is defined by $A(\vec{n})=1-3 \varepsilon-4 \varepsilon\left(\phi_{x}^{4}+\phi_{y}^{4}+\phi_{z}^{4}\right) /\left(\phi_{x}^{2}+\phi_{y}^{2}+\phi_{z}^{2}\right)^{2}$. To simulate multidendrite growth with different crystalline orientations, each dendrite was assigned a local coordinate system $\left(x_{0}, y_{0}, z_{0}\right)$ and individual orientation angle by three Euler angles $(\alpha, \beta, \gamma)$. An extra orientation field is defined for each cell, and the cell orientation is equal to the orientation of the surrounding cell which possesses the largest fraction solid.

\subsection{Multiscale Coupling Model}

To improve computational efficiency, different grid resolutions in different space scales were used, which is shown in Figure 2. The coarse grids were used for heat transfer simulation, the finer grids 
were used for CA-based grain growth simulation, and the finest grids were used for phase-field-based dendrite growth simulation. During each time increment, the temperature in each CA and phase-field cell was calculated from the surrounding larger grids by using the interpolation method, which is shown in Figure 2a. In contrast, the temperature in the phase-field cells were taken as being uniform and equal to the larger CA cell. Figure $2 b, c$ show the modified CA-based cell capture algorithm and the phase-field model, respectively. In the modified CA algorithm, the effect of grain orientation was considered, and additional cells were captured along the grain orientation direction. In the phase-field model, the solid and liquid phases were defined by a scalar field. The temporal evolution of each phase was controlled by the variation of local free energy, which is a function of temperature, concentration, and phase fraction.

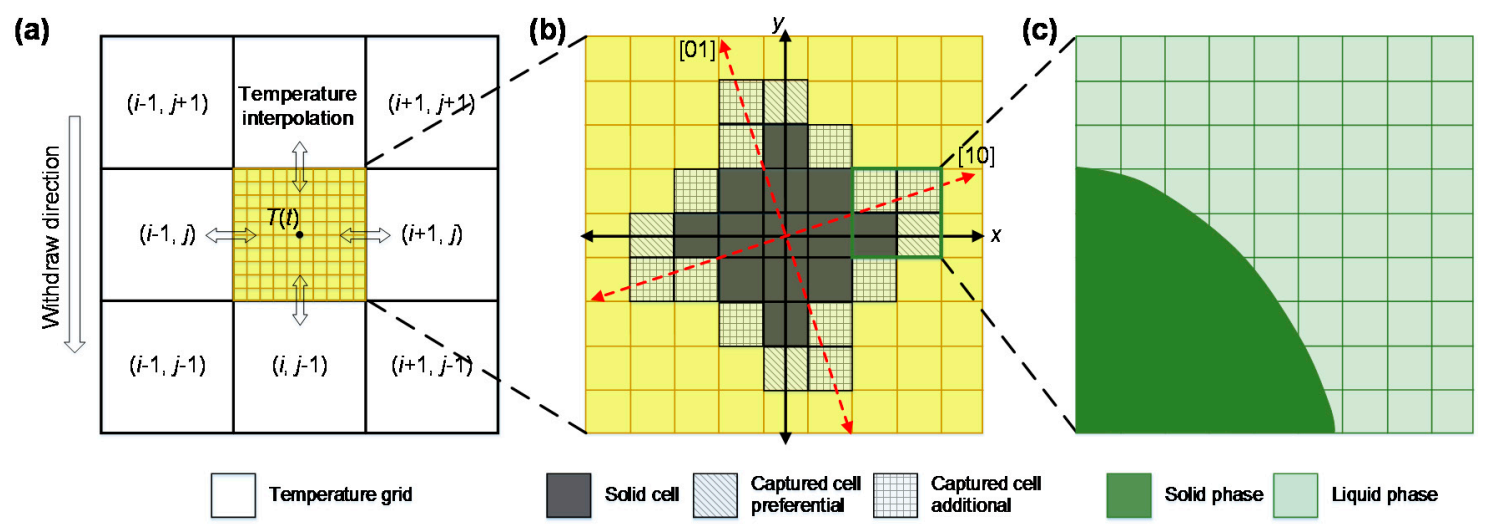

Figure 2. Schematic of coupling calculation between the macro and micro scales: (a) temperature interpolation from coarse macro grids to fine CA cells; (b) modified CA-based cell capture algorithm for grain growth simulation; and (c) the smallest phase-field grids for micro scale dendrite growth simulation.

\section{Results and Discussion}

\subsection{Simulation Parameters}

The temperature field evolution and grain growth of an aero-engine blade and an industrial gas turbine (IGT) blade during LMC and HRS directional solidification processes were simulated and analyzed. The alloy used was DD6 (Ni-4.3Cr-9Co-2Mo-8W-7.5Ta-2Re-0.5Nb-5.6Al-0.1Hf, wt \%), which is a low Re-bearing, second-generation, Ni-based, single crystal superalloy. The macroscopic simulations of the temperature field and grain growth during superalloy directional solidification were performed using the developed CA-FD method. Furthermore, the microscopic simulations of the dendrite growth were performed using the phase-field method. In all simulations, the solidification of the multicomponent DD6 superalloy was simplified as a pseudo-binary Ni-X alloy. The physical and thermodynamic parameters of the alloy were calculated using PANDAT software (Software version: 2013.2.1, 8401 Greenway Blvd. Suite 248, Middleton, WI, USA) and listed in Table 1.

In calculating the temperature field in HRS and LMC solidification processes, the initial and boundary conditions are vital for reflecting the actual heat transfer process. The main parameters used in the heat transfer calculation during typical HRS/LMC processes are listed in Table 2. The initial and boundary conditions were applied to the furnace, casting, mold, chill plate, and tin bath. During the withdrawal process, the radiation view factors (for HRS and LMC) and the mold/tin boundary (for LMC) were updated at intervals of $0.5 \mathrm{~mm}$ for the withdrawal distance. The fluid flow inside the casting and the tin bath were not considered in the current study. Otherwise, there would be a significant addition in computational cost for the simulations. 
Table 1. Physical and thermodynamic parameters of the pseudo-binary Ni-X superalloy [21,22].

\begin{tabular}{ccc}
\hline Parameters and Symbols & Unit & Values \\
\hline Liquidus temperature, $T_{\mathrm{L}}$ & $\mathrm{K}$ & 1672 \\
Solidus temperature, $T_{\mathrm{S}}$ & $\mathrm{K}$ & 1615 \\
Thermal conductivity, $\lambda$ & $\mathrm{W} \cdot \mathrm{m}^{-1} \cdot \mathrm{K}^{-1}$ & 33.2 \\
Density, $\rho$ & $\mathrm{kg} \cdot \mathrm{m}^{-3}$ & 8780 \\
Specific heat, $c_{\mathrm{p}}$ & $\mathrm{J} \cdot \mathrm{kg}^{-1} \cdot \mathrm{K}^{-1}$ & 773 \\
Latent heat, $\Delta H$ & $\mathrm{~J} \cdot \mathrm{kg}^{-1}$ & $9.9 \times 10^{4}$ \\
Interface energy, $\sigma$ & $\mathrm{J} \cdot \mathrm{m}^{-2}$ & 0.161 \\
Initial concentration of $X, c_{\infty}$ & $\mathrm{wt} \%$ & 39.006 \\
Partition coefficient, $k$ & - & 0.788 \\
Liquidus slope, $m_{l}$ & $\mathrm{~K} \cdot \mathrm{wt} \%{ }^{-1}$ & -3.95 \\
Gibbs-Thomson coefficient, $\Gamma$ & $\mathrm{K} \cdot \mathrm{m}$ & $3.65 \times 10^{-7}$ \\
Anisotropy strength, $\varepsilon$ & - & 0.02 \\
Liquid solute diffusivity, $D_{l}$ & $\mathrm{~m}^{2} \cdot \mathrm{s}^{-1}$ & $3.6 \times 10^{-9}$ \\
\hline
\end{tabular}

Table 2. The initial and boundary conditions during directional solidification.

\begin{tabular}{ccc}
\hline Parameters & Unit & Values (HRS/LMC) \\
\hline Temperature of the heating zone & $\mathrm{K}$ & 1773 \\
Temperature of the cooling zone/tin bath & $\mathrm{K}$ & $298 / 523$ \\
Temperature of the chill & $\mathrm{K}$ & $298 / 523$ \\
Heat transfer coefficient between mold and tin & $\mathrm{W} \cdot \mathrm{m}^{-2} \cdot \mathrm{K}^{-1}$ & $-/ 4500[11]$ \\
Heat transfer coefficient between metal and chill & $\mathrm{W} \cdot \mathrm{m}^{-2} \cdot \mathrm{K}^{-1}$ & $10,000[28]$ \\
Heat transfer coefficient between metal and mold & $\mathrm{W} \cdot \mathrm{m}^{-2} \cdot \mathrm{K}^{-1}$ & $600[29]$ \\
Thermal conductivity of chill & $\mathrm{W} \cdot \mathrm{m}^{-1} \cdot \mathrm{K}^{-1}$ & $401 / 14[10]$ \\
Thermal conductivity of mold & $\mathrm{W} \cdot \mathrm{m}^{-1} \cdot \mathrm{K}^{-1}$ & $2.5[11]$ \\
Thermal conductivity of liquid tin & $\mathrm{W} \cdot \mathrm{m}^{-1} \cdot \mathrm{K}^{-1}$ & $-/ 34$ \\
Emissivity of the metal and furnace & - & $0.8[28]$ \\
Emissivity of the mold & - & 0.6 \\
\hline
\end{tabular}

\subsection{Temperature and Mushy Zone Evolution}

The temperature field and mushy zone evolution of the single crystal aero-engine blade during HRS and LMC processes are shown in Figures 3 and 4, respectively. The temperature range in the color bar is from solidus to liquidus and thus, the shape of the mushy zone can be seen clearly. The withdrawal rate was $3 \mathrm{~mm} / \mathrm{min}$ for typical HRS process and $9 \mathrm{~mm} / \mathrm{min}$ for typical LMC process. During the initial solidification stage, the temperature field in HRS and LMC processes both exhibited a high thermal gradient of about $40 \mathrm{~K} / \mathrm{mm}$ due to the high cooling efficiency of the chill plate. As the withdrawal process continued, the thermal gradient reduced rapidly in the HRS process and led to an increase in mushy zone width. In contrast, the LMC process still maintained a high temperature gradient due to the strong cooling effect of the tin bath. The typical thermal gradients of the HRS and LMC processes in a steady solidification state were $2 \mathrm{~K} / \mathrm{mm}$ and $8 \mathrm{~K} / \mathrm{mm}$, respectively, according to the simulations, which is in accordance with previous experimental results [30]. The high thermal gradient in the LMC process was beneficial for producing single crystal blades with reduced primary dendritic spacing and refined $\gamma^{\prime}$ phase morphology. 


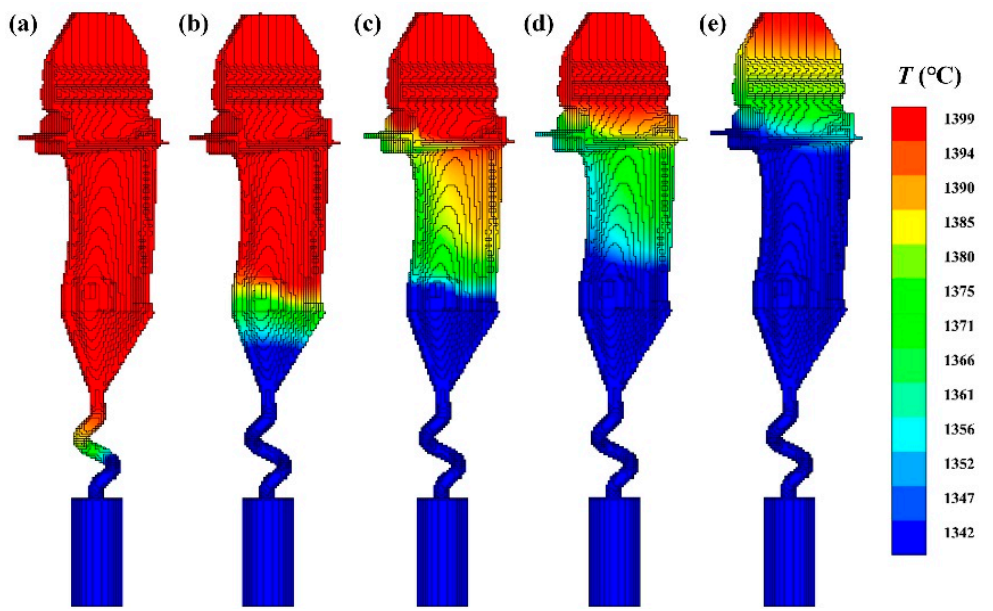

Figure 3. The temperature distribution and mushy zone evolution by the HRS technique at a constant withdrawal rate of $3 \mathrm{~mm} / \mathrm{min}$ : (a) $t=241 \mathrm{~s}, f_{\mathrm{s}}=30 \%$; (b) $t=2028 \mathrm{~s}, f_{\mathrm{s}}=40 \%$; (c) $t=2411 \mathrm{~s}, f_{\mathrm{s}}=50 \%$; (d) $t=2632 \mathrm{~s}, f_{\mathrm{s}}=60 \%$; and (e) $t=2987 \mathrm{~s}, f_{\mathrm{s}}=80 \%$. In this figure, $f_{\mathrm{s}}$ stands for fraction solid.

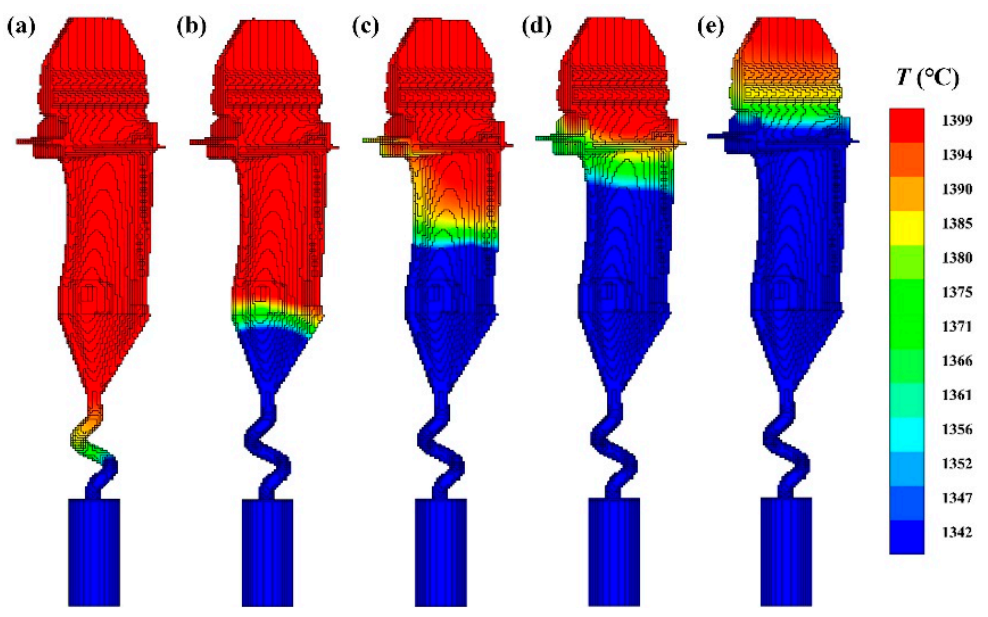

Figure 4. The temperature distribution and mushy zone evolution by the LMC technique at a constant withdrawal rate of $8 \mathrm{~mm} / \mathrm{min}$ : (a) $t=205 \mathrm{~s}, f_{\mathrm{s}}=30 \%$; (b) $t=689 \mathrm{~s}, f_{\mathrm{s}}=40 \%$; (c) $t=910 \mathrm{~s}, f_{\mathrm{s}}=50 \%$; (d) $t=1031 \mathrm{~s}, f_{\mathrm{s}}=60 \%$; and (e) $t=1193 \mathrm{~s}, f_{\mathrm{s}}=80 \%$. In this figure, $f_{\mathrm{s}}$ stands for fraction solid.

The temperature evolution of large IGT blades in LMC directional solidification was also investigated. To improve the high-temperature mechanical properties of the polycrystalline blade, the grains and grain boundaries are required to be parallel with the stress direction, which is also the heat flow direction during solidification. Therefore, a flat mushy zone morphology is favorable in producing the large IGT blades. In the current investigation, two types of withdrawal rates were applied: one is a constant value of $6 \mathrm{~mm} / \mathrm{min}$ and the other is an optimized location dependent varying withdraw rate, which is shown in Figure 5.

Figure 6 shows the mushy zone evolution with a constant (a1-a5) and varying withdrawal rate (b1-b5) that was applied to the industrial gas turbine blade during the LMC process. In the early stage of the LMC process with a constant withdrawal rate, the convex shape of the mushy zone can be observed (Figure 6(a1)). The initial convex mushy zone morphology can be ascribed to the low temperature and fine thermal conductivity of the chill. However, this convex shape of the mushy zone can cause the divergence of the columnar grains [10], which is harmful to the blade's mechanical integrity. As the withdrawal process continued, the mushy zone was transformed to concave shape (Figure 6(a2-a5)) due to the weakened heat transfer rate between the casting and chill. This heat 
transfer was mainly controlled by the conduction between casting and mold. Similarly, this convex shape of mushy zone is not favorable because it will lead to misaligned grains. By altering the withdrawal velocity, it is feasible to control the location of solid-liquid interface and the local cooling condition, thus changing the mushy zone morphology. Elliot [10] and Yan [23] have investigated the effects of withdrawal velocity on the solid/liquid curvature, and found that small withdrawal velocity will lead to a convex shape of the mushy zone and large withdrawal velocity will result in a concave shape of the mushy zone. As shown in Figure 6(b1-b5), the optimized varying withdrawal velocity has changed the curved mushy zone to a flat shape, which is beneficial for the growth of well-aligned columnar grains in the IGT blade.

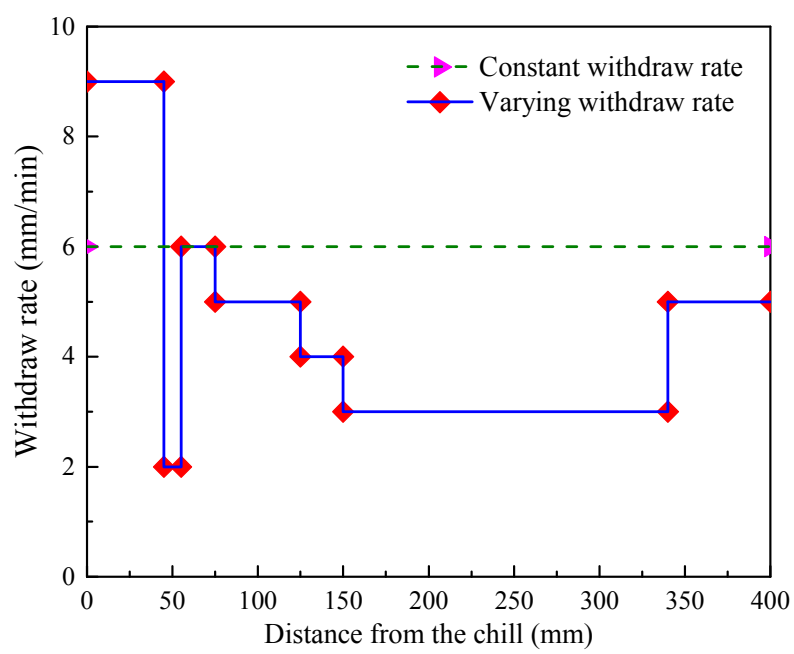

Figure 5. The constant and varying withdrawal rates that are applied to an IGT blade during the LMC process.
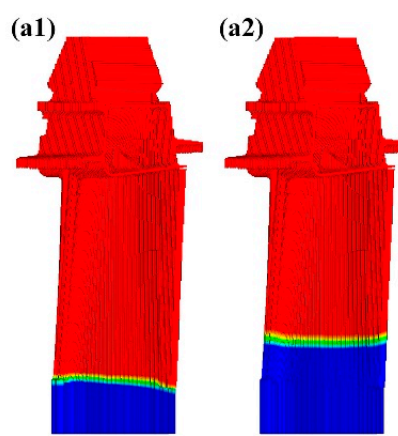

(a3)
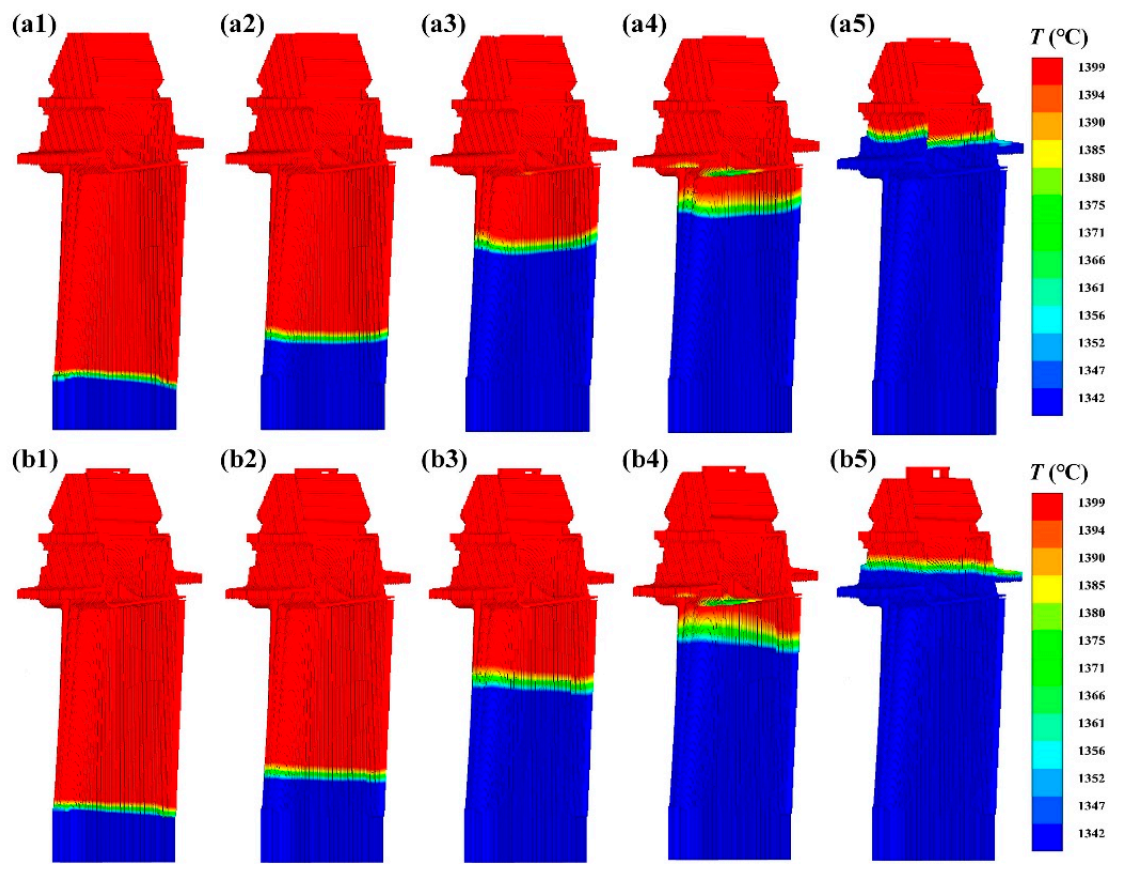

(b3)

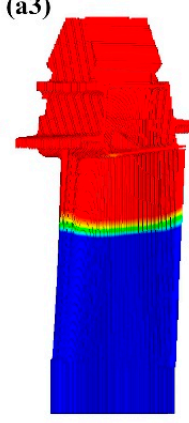

(b4)
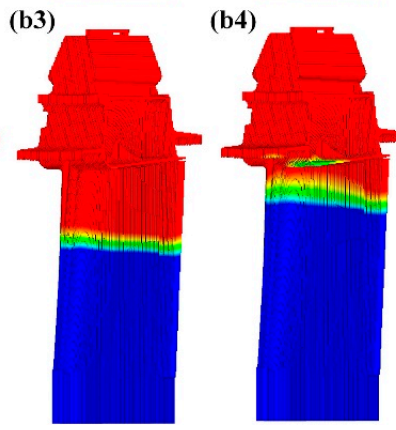

(b5)

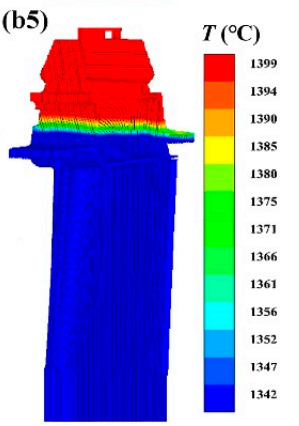

Figure 6. The mushy zone evolution with constant (a1-a5) and varying withdrawal rate (b1-b5) that are applied to the industrial gas turbine blade during the LMC process. The fraction solid in each figure is: $(\mathbf{a} 1, \mathbf{b} 1) f_{\mathrm{s}}=9 \% ;(\mathbf{a} 2, \mathbf{b} 2) f_{\mathrm{s}}=13 \% ;(\mathbf{a} 3, \mathbf{b 3}) f_{\mathrm{s}}=24 \% ;(\mathbf{a} 4, \mathbf{b} 4) f_{\mathrm{s}}=31 \%$; and $(\mathbf{a} 5, \mathbf{b} 5) f_{\mathrm{s}}=67 \%$. 


\subsection{Grain Structure Evolution}

The grain growth in directional solidified single crystal aero-engine blades and large IGT blades was investigated using the developed CA-FD method, and the simulation results were compared with the experimental results. Figure 7 shows the simulated and experimental results of the grain structure in a single crystal blade during HRS (a1-a3) and LMC (b1-b3) processes. The start block and the spiral selector part were shown in the enlarged view, and the different colors mean grains with different angles of $<001>$ crystalline orientation with respect to the heat flow direction. The HRS and LMC solidification techniques were both able to produce single crystal blades by using the spiral selector according to the simulation and experimental results. At the bottom of the start block, a large number of nuclei can be found and these nuclei were formed under the strong cooling effect of the chill plate. After nucleation, grain competitive growth happens and grains with small orientation angles can overgrow the grains with large orientation angles. At the top of the starter block, only a few grains survive and grow into the spiral part. Finally, only one grain will occupy the spiral part due to the special designed geometry. The columnar grains were coarser and sparser under the LMC solidification process compared with the HRS results because the water-cooling copper chill plate in the HRS process had a better initial cooling effect compared with the stainless steel chill plate used in the LMC process.

(a1)

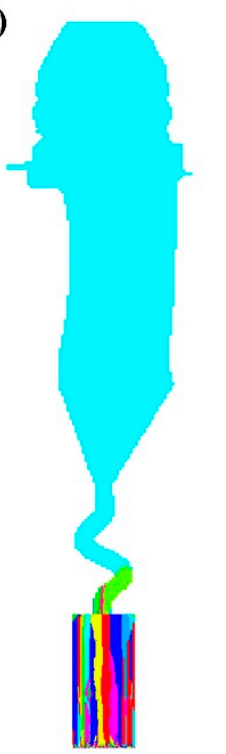

(a2)

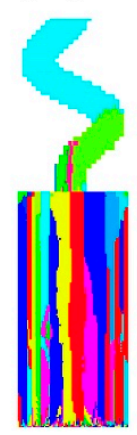

(a3)

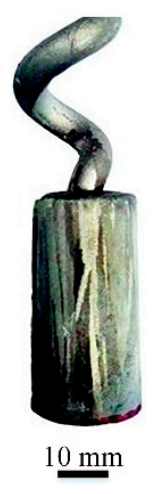

(b1)

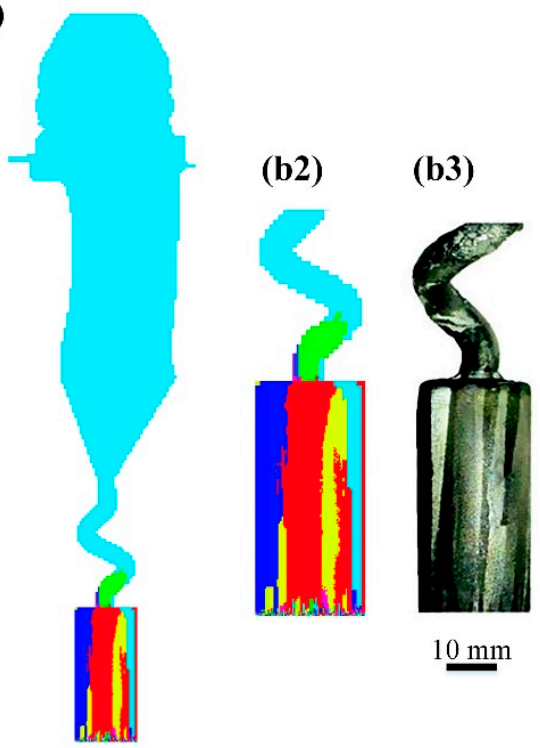

Figure 7. The simulated and experimental results of grain structure in a single crystal blade during HRS (a1-a3) and LMC (b1-b3) processes.

Figure 8 shows the simulated (a) front and (c) back and experimental results as well as (b) front and (d) back of the grain structure in the LMC directional solidified industrial gas turbine blade. After being nucleated on the chill plate, columnar grains quickly developed and the grains with large orientation angles were eliminated through competitive growth. After applying the varying withdrawal velocity on the IGT blade, the mushy zone morphology became flat, and the resulting grains were almost parallel with the withdrawal direction. The simulated grain structures were generally in agreement with the experimental results, which demonstrate the ability and good precision of the developed CA-FD algorithm. However, there were still some broken grains in the front side of the blade, and some stray grains were found to be nucleated on the platform of the blade. These grains are harmful to the high-temperature mechanical property of the blade. In addition, further investigations should be done to inhibit the occurrence of these grain defects. 
(a)

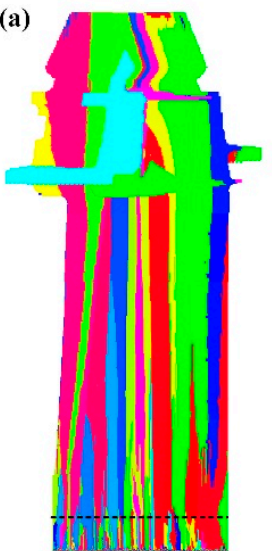

(b)

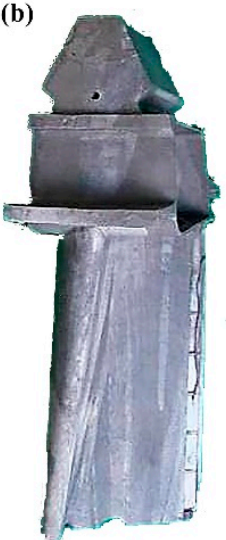

(c)

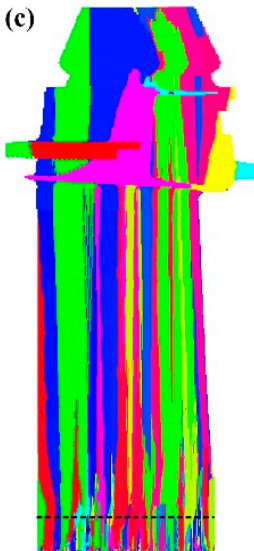

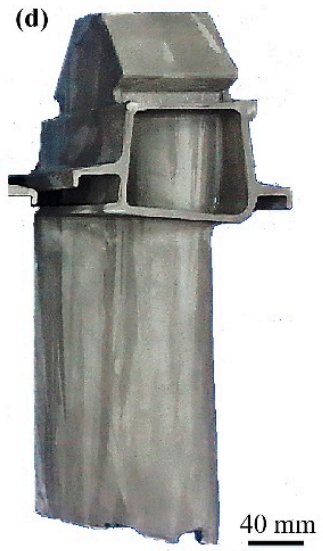

Figure 8. The simulated $(\mathbf{a}, \mathbf{c})$ and experimental results $(\mathbf{b}, \mathbf{d})$ of grain structures in LMC directional solidified industrial gas turbine blade. The grains below the black lines in the simulation results will be cut off in the following machining process.

\subsection{Dendrite Growth}

\subsubsection{Two-Dimensional Dendrite Growth}

Dendrites are one of the most important microstructure features in superalloy castings. An understanding of the dendrite growth in directional solidified superalloy blades is vital for improving the performance. However, the phase-field simulation of dendrite growth requires a large amount of computation and it is not possible to simulate the growth of all dendrites in the superalloy blades. Therefore, the two-dimensional dendrite growth in different sections of the single crystal blade were simulated as an example. Figure 9 shows the simulation and experimental results of the grain (a) and dendrite morphologies $(b-d)$ in the single crystal blade during the HRS process as described above. The dendrites exhibit a typical cross shape and some of the dendrites have secondary arms. The simulated dendrite morphology was generally in agreement with experimental results.

(a)

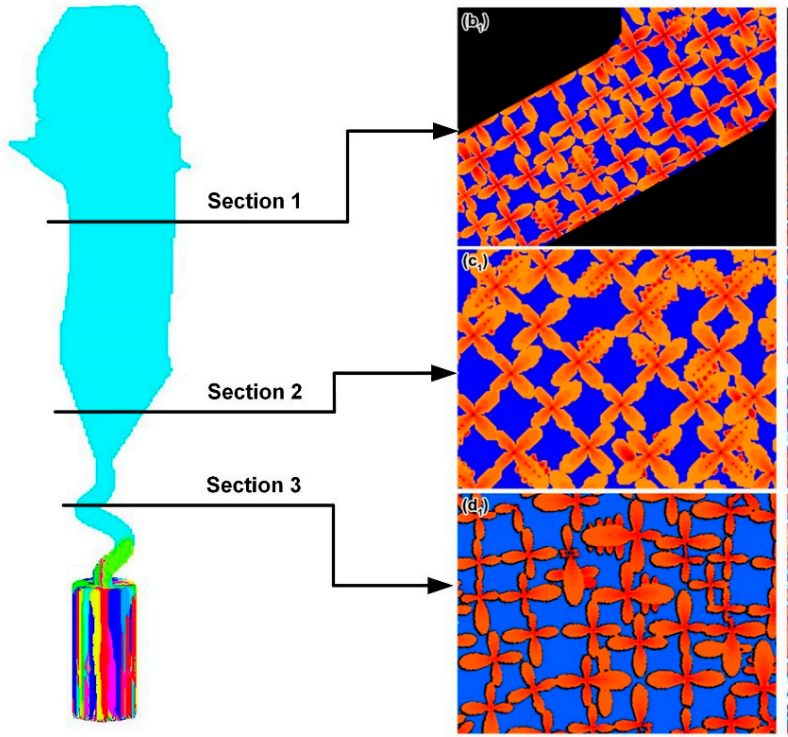

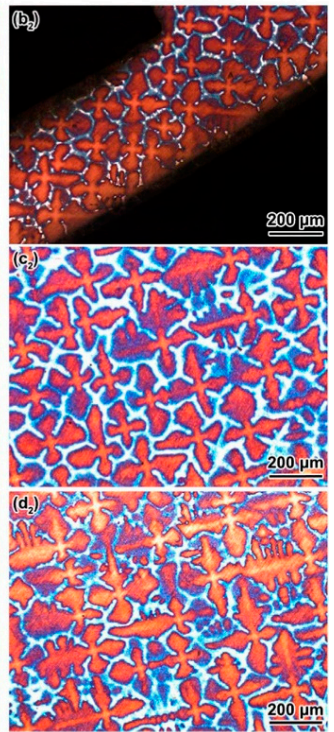

Figure 9. Simulation and experimental results of the grain and dendrite morphologies: (a) grain structure of the single crystal blade under HRS process; $(\mathbf{b} 1, \mathbf{b} 2)$ dendrite morphology in the hollow part; (c1,c2) dendrite morphology in the lower part; and $(\mathbf{d} 1, \mathbf{d} 2)$ dendrite morphology in the spiral selector part. 


\subsubsection{Three-Dimensional Dendrite Growth}

The 3D single crystal dendrite growth during HRS and LMC processes was also investigated using the phase-field method. The withdrawal velocity was set to $3 \mathrm{~mm} / \mathrm{min}$ and $9 \mathrm{~mm} / \mathrm{min}$, and the temperature gradient was set to $5 \mathrm{~K} / \mathrm{mm}$ and $15 \mathrm{~K} / \mathrm{mm}$ for the typical HRS and LMC solidification processes, respectively. A previously developed GPU-based parallel computing scheme [21] was used to accelerate the phase-field simulation. To explore a long withdrawal distance in $z$ direction, a moving-frame algorithm was used. The alloy parameters are listed in Table 1. At the initial time, the bottom of the computational domain was set with a slice of solid (FCC) phase, and the dendrites were developed from the planar interface by Mullins-Sekerka instability [31]. During the simulations, periodic boundary conditions were applied in $x$ and $y$ directions, while a nonflux boundary condition was applied in the $z$ direction. The total time steps of each phase-field simulation was $2 \times 10^{6}$, which corresponds to a physical solidification time of $100 \mathrm{~s}$.

Figure 10 shows the simulation results of dendrite morphology under HRS and LMC solidification processes at different solidification times. At the initial growth stage, hundreds of perturbations were developed through the planar instability and some of these perturbations could have grown into dendrite arms with a typical cross-shaped pattern. Afterwards, the dendrite competitive growth started, many dendrites were eliminated, and the primary dendrite arms were able to adjust their position through lateral movements. After a long withdrawal distance, the dendrite steady growth stage was reached for both HRS and LMC directional solidification processes, with the dendrite morphology and primary dendrite arm spacing remaining almost unchanged. The primary dendrite arms under steady state conditions were coarser and sparser in the HRS process compared with the dendrite arms in the LMC process, which means that the solidification conditions have a significant effect on the dendrite morphology and dendritic spacing. The previous experimental results [10,32] also revealed reduced dendritic spacing in the LMC process compared with the HRS process. Figure 11 shows the top view of the (a) SEM image and (b) phase-field simulation results of the primary dendrite arms in the HRS directional solidification process. Furthermore, the simulated dendrite morphology was generally in agreement with the experimental results.
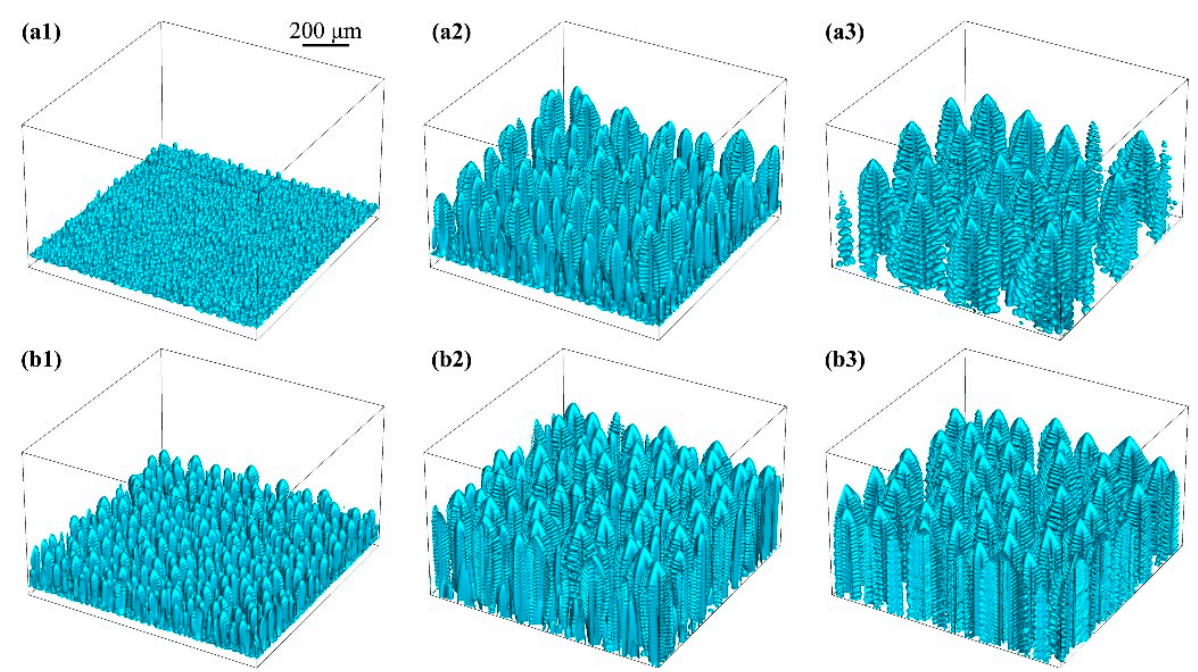

Figure 10. The dendrite morphology under HRS solidification process at time of (a1) $t=10 \mathrm{~s},(\mathbf{a} 2) t=15 \mathrm{~s}$, (a3) $t=100 \mathrm{~s}$; and under LMC solidification process at time of (b1) $t=5 \mathrm{~s},(\mathbf{b} 2) t=7.5 \mathrm{~s}$ and (b3) $t=100 \mathrm{~s}$. 

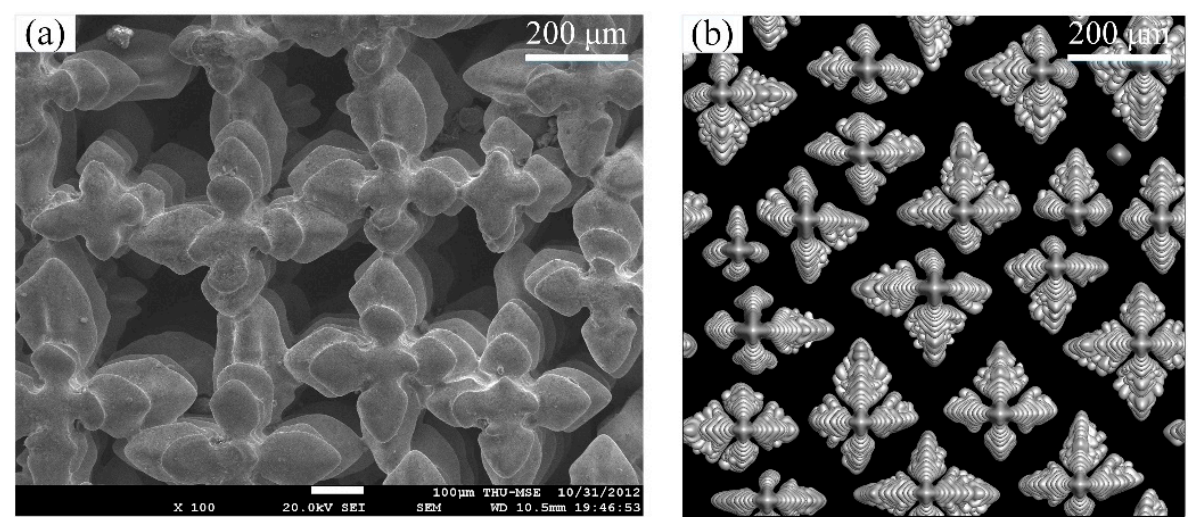

Figure 11. Top view of the (a) SEM image and (b) phase-field simulation result of the primary dendrite arms in HRS directional solidification process.

\section{Conclusions}

The 3D CA-FD method and phase-field method were used to simulate solidification and microstructure evolution on multiple multiscale models. The temperature field and mushy zone evolution in directional solidified blades under HRS and LMC processes were calculated and analyzed. Furthermore, the simulated grain structures were compared with experimental results. The dendrite growth in typical HRS and LMC directional solidification processes was also investigated. The main conclusions are listed as follows.

(1) The developed multiscale model, which combined the 3D CA-FD method and phase-field method, can be used to calculate complex heat transfer processes, grain growth, and dendrite growth in superalloy directional solidification.

(2) There is a larger temperature gradient and narrower mushy zone in the LMC process compared with the HRS process, while there is capacity for a larger withdrawal velocity in the LMC process. By applying varying withdrawal velocity, it is possible to obtain a flat mushy zone shape, which is favored for the growth of well-aligned grains.

(3) The simulated structure of grains in HRS and LMC processes is in agreement with experimental results. The columnar grains in the starter block part in the HRS process are denser than those in the LMC process due to the better cooling effect of the water cooling copper plate.

(4) Phase-field simulations have been performed to investigate the effect of solidification conditions on dendrite morphology and dendritic spacing. The simulation results were in agreement with experimental results as they both found that the dendrite morphology is coarser and the primary dendritic spacing is larger in the HRS process compared with those in the LMC process.

Author Contributions: Q.X. and C.Y. wrote the paper; C.Y. and N.T. did the simulations; H.Z., X.Y., and N.T. performed the experiments; Q.X. and B.L. revised the paper.

Funding: This research was funded by the National Science and Technology Major Project (No. 2017ZX04014001), National Key Research and Development Program of China (2017YFB0701503), and the National Natural Science Foundation of China (No. 51374137).

Conflicts of Interest: The authors declare no conflict of interest.

\section{References}

1. Bridgman, P.W. Crystals and Their Manufacture. U.S. Patent US1793672A, 24 February 1931.

2. Versnyder, F.I.; Shank, M.E. The development of columnar grain and single crystal high temperature materials through directional solidification. Mater. Sci. Eng. 1970, 6, 213-247. [CrossRef]

3. Giamei, A.F.; Tschinkel, J.G. Liquid metal cooling: A new solidification technique. Metall. Trans. A 1976, 7 , 1427-1434. [CrossRef] 
4. Liu, L.; Huang, T.; Zhang, J.; Fu, H. Microstructure and stress rupture properties of single crystal superalloy cmsx-2 under high thermal gradient directional solidification. Mater. Lett. 2007, 61, 227-230. [CrossRef]

5. Liu, C.; Shen, J.; Zhang, J.; Lou, L. Effect of withdrawal rates on microstructure and creep strength of a single crystal superalloy processed by lmc. J. Mater. Sci. Technol. 2010, 26, 306-310. [CrossRef]

6. Xu, Y.; Sun, W.; Dai, W.; Hu, C.; Liu, X.; Zhang, W. Experimental and numerical modeling of the stress rupture behavior of nickel-based single crystal superalloys subject to multi-row film cooling holes. Metals 2017, 7, 340. [CrossRef]

7. Xu, C.; Liu, F.; Huang, L.; Jiang, L. Dependence of creep performance and microstructure evolution on solution cooling rate in a polycrystalline superalloy. Metals 2018, 8, 4. [CrossRef]

8. Gandin, C.A.; Desbiolles, J.L.; Rappaz, M.; Thevoz, P. A three-dimensional cellular automation-finite element model for the prediction of solidification grain structures. Metall. Mater. Trans. A 1999, 30, 3153-3165. [CrossRef]

9. Kermanpur, A.; Rappaz, M.; Varahram, N.; Davami, P. Thermal and grain-structure simulation in a land-based turbine blade directionally solidified with the liquid metal cooling process. Metall. Mater. Trans. B 2000, 31, 1293-1304. [CrossRef]

10. Elliott, A.J.; Pollock, T.M.; Tin, S.; King, W.T.; Huang, S.C.; Gigliotti, M.F.X. Directional solidification of large superalloy castings with radiation and liquid-metal cooling: A comparative assessment. Metall. Mater. Trans. A 2004, 35, 3221-3231. [CrossRef]

11. Elliott, A.J.; Pollock, T.M. Thermal analysis of the bridgman and liquid-metal-cooled directional solidification investment casting processes. Metall. Mater. Trans. A 2007, 38, 871-882. [CrossRef]

12. Zhang, H.; Xu, Q.Y.; Tang, N.; Pan, D.; Liu, B.C. Numerical simulation of microstructure evolution during directional solidification process in directional solidified (ds) turbine blades. Sci. China Technol. Sci. 2011, 54, 3191-3202. [CrossRef]

13. Xu, Q.Y.; Liu, B.C.; Dong, P.; Jing, Y. Progress on modeling and simulation of directional solidification of superalloy turbine blade casting. China Foundry 2012, 62, 69-77.

14. Zhang, H.; Xu, Q.Y. Simulation and experimental studies on grain selection and structure design of the spiral selector for casting single crystal ni-based superalloy. Materials 2017, 10, 1236. [CrossRef] [PubMed]

15. Wang, W.; Kermanpur, A.; Lee, P.D.; McLean, M. Simulation of dendritic growth in the platform region of single crystal superalloy turbine blades. J. Mater. Sci. 2003, 38, 4385-4391. [CrossRef]

16. Kim, S.G.; Kim, W.T.; Suzuki, T. Phase-field model for binary alloys. Phys. Rev. E 1999, 60, 7186-7197. [CrossRef]

17. Echebarria, B.; Folch, R.; Karma, A.; Plapp, M. Quantitative phase-field model of alloy solidification. Phys. Rev. E 2004, 70, 061604. [CrossRef] [PubMed]

18. Eiken, J.; Böttger, B.; Steinbach, I. Multiphase-field approach for multicomponent alloys with extrapolation scheme for numerical application. Phys. Rev. E 2006, 73, 066122. [CrossRef] [PubMed]

19. Yang, C.; Xu, Q.Y.; Liu, B.C. Primary dendrite spacing selection during directional solidification of multicomponent nickel-based superalloy: Multiphase-field study. J. Mater. Sci. 2018, 53, 9755-9770. [CrossRef]

20. Takaki, T.; Sakane, S.; Ohno, M.; Shibuta, Y.; Shimokawabe, T.; Aoki, T. Primary arm array during directional solidification of a single-crystal binary alloy: Large-scale phase-field study. Acta Mater. 2016, 118, 230-243. [CrossRef]

21. Yang, C.; Xu, Q.Y.; Liu, B.C. Gpu-accelerated three-dimensional phase-field simulation of dendrite growth in a nickel-based superalloy. Comput. Mater. Sci. 2017, 136, 133-143. [CrossRef]

22. Zhang, H.; Xu, Q.Y.; Shi, Z.X.; Liu, B.C. Numerical simulation of dendrite grain growth of dd6 superalloy during directional solidification process. Acta Metall. Sin. 2014, 50, 345-354.

23. Yan, X.W.; Tang, N.; Liu, X.F.; Shui, G.Y.; Xu, Q.Y.; Liu, B.C. Modeling and simulation of directional solidification by lmc process for nickel base superalloy casting. Acta Metall. Sin. 2015, 1288-1296.

24. Zhang, H.; Xu, Q.; Liu, B. Numerical simulation and optimization of directional solidification process of single crystal superalloy casting. Materials 2014, 7, 1625-1639. [CrossRef] [PubMed]

25. Rappaz, M.; Gandin, C.A. Probabilistic modelling of microstructure formation in solidification processes. Acta Metall. Mater. 1993, 41, 345-360. [CrossRef]

26. Kurz, W.; Giovanola, B.; Trivedi, R. Theory of microstructural development during rapid solidification. Acta Metall. 1986, 34, 823-830. [CrossRef] 
27. Diepers, H.-J.; Ma, D.; Steinbach, I. History effects during the selection of primary dendrite spacing. Comparison of phase-field simulations with experimental observations. J. Cryst. Growth 2002, 237, 149-153. [CrossRef]

28. Franke, M.M.; Hilbinger, R.M.; Lohmüller, A.; Singer, R.F. The effect of liquid metal cooling on thermal gradients in directional solidification of superalloys: Thermal analysis. J. Mater. Process. Technol. 2013, 213, 2081-2088. [CrossRef]

29. Konrad, C.H.; Brunner, M.; Kyrgyzbaev, K.; Völkl, R.; Glatzel, U. Determination of heat transfer coefficient and ceramic mold material parameters for alloy in7381c investment castings. J. Mater. Process. Technol. 2011, 211, 181-186. [CrossRef]

30. Carter, P.; Cox, D.C.; Gandin, C.A.; Reed, R.C. Process modelling of grain selection during the solidification of single crystal superalloy castings. Mater. Sci. Eng. A 2000, 280, 233-246. [CrossRef]

31. Mullins, W.W.; Sekerka, R.F. Stability of a planar interface during solidification of a dilute binary alloy a2-Pelcé, pierre. In Dynamics of Curved Fronts; Academic Press: San Diego, CA, USA, 1988; pp. 345-352.

32. Yan, X.W.; Xu, Q.Y.; Liu, B.C. Numerical simulation and experimental casting of nickel-based single-crystal superalloys by hrs and lmc directional solidification processes. High Temp. Mater Process. 2017, 36, 327-337. [CrossRef]

(C) 2018 by the authors. Licensee MDPI, Basel, Switzerland. This article is an open access article distributed under the terms and conditions of the Creative Commons Attribution (CC BY) license (http://creativecommons.org/licenses/by/4.0/). 